\title{
Structures of High Density Molecular Fluids
}

B. J. Baer*, H. Cynn, V. lota, and C.-S. Yoo

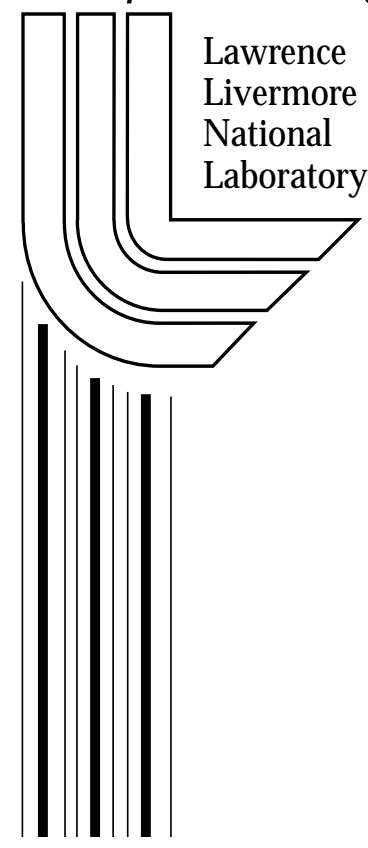

February 01, 2002 


\section{DISCLAIMER}

This document was prepared as an account of work sponsored by an agency of the United States Government. Neither the United States Government nor the University of California nor any of their employees, makes any warranty, express or implied, or assumes any legal liability or responsibility for the accuracy, completeness, or usefulness of any information, apparatus, product, or process disclosed, or represents that its use would not infringe privately owned rights. Reference herein to any specific commercial product, process, or service by trade name, trademark, manufacturer, or otherwise, does not necessarily constitute or imply its endorsement, recommendation, or favoring by the United States Government or the University of California. The views and opinions of authors expressed herein do not necessarily state or reflect those of the United States Government or the University of California, and shall not be used for advertising or product endorsement purposes.

This work was performed under the auspices of the U. S. Department of Energy by the University of California, Lawrence Livermore National Laboratory under Contract No. W-7405-Eng-48.

This report has been reproduced

directly from the best available copy.

A vailable to DOE and DOE contractors from the

Office of Scientific and Technical Information

P.O. Box 62, Oak Ridge, TN 37831

Prices available from (423) 576-8401

http:/ / apollo.osti.gov/ bridge/

A vailable to the public from the National Technical Information Service

U.S. Department of Commerce 5285 Port Royal Rd.,

Springfield, VA 22161

Lawrence

Livermore

National

Laboratory

http:/ / www.ntis.gov/

OR

Lawrence Livermore National Laboratory

Technical Information Department's Digital Library

http:/ / www.Ilnl.gov/ tid/ Library.html 


\section{Objective:}

The goal of this proposal is to develop an in-situ probe for high density molecular fluids. We will, therefore, use Coherent Anti-Stokes Raman Spectroscopy (CARS) applied to laser heated samples in a diamond-anvil cell (DAC) to investigate molecular fluids at simultaneous conditions of high temperatures $(\mathrm{T}>2000 \mathrm{~K})$ and high pressures $(\mathrm{P}>10 \mathrm{GPa}$.) Temperatures sufficient to populate vibrational levels above the ground state will allow the vibrational potential to be mapped by CARS. A system capable of heating and probing these samples will be constructed. Furthermore, the techniques that enable a sample to be sufficiently heated and probed while held at static high pressure in a diamond-anvil-cell will be developed. This will be an in-situ investigation of simple molecules under conditions relevant to the study of detonation chemistry and the Jovain planet interiors using state of the art non-linear spectroscopy, diamond-anvil-cells, and laser heating technology.

\section{Introduction:}

Diamond-anvil-cells (DACs) have been used for several decades to generate high pressures. However, only recently have more than a handful of experiments been done at temperatures well above $1000 \mathrm{~K}$ in a DAC. Most of these experiments usually involve x-ray crystallography or the visual observation for melting. For samples at the proposed experimental conditions, detailed spectroscopic investigation has proven very difficult. Many of the classical methods of IR and Raman spectroscopy simply will not work well when the sample is incandescent. Here we outline a technique that will permit the investigation of Raman active vibrations in molecules that are held under static conditions at high temperatures and pressures. Such studies have important implications for HE-detonation, planetary models, and novel materials syntheses.

Two problems must be overcome in order to make a DAC a useful tool for the spectroscopic study of high density fluids at high temperatures. The first is generating high temperatures with a small thermal gradient despite the fact that diamond has the highest known thermal conductivity. The second is utilizing a spectroscopic technique sufficiently robust that will work in a DAC and overcome the intense Planck radiation of a sample above $2000 \mathrm{~K}$. 


\section{Experimental Technique and Development:}

High pressures are generated on microscopic samples in a device known as a diamond-anvilcell (DAC.) Brilliant cut diamonds are modified to have a small flat area, known as a culet, instead of converging to a faceted point. Two of these anvils are opposed and aligned so that the culets come together. In this way, a force on the larger flat surface, known as the table, is amplified when transmitted to the culets. A thin piece of metal acts a gasket to prevent sample extrusion from between the culets. This metal piece has an aperture drilled into it that is about one third the size of the culets. Typically the culets are about 300 microns in diameter. Pressures are determined by placing a small chip of ruby or $\mathrm{Sm}^{2+}: \mathrm{SrB}_{4} \mathrm{O}_{7}$ along with the sample and measuring its laser induced emission. The luminescence of these crystals has been previously calibrated for temperature and pressure effects. An oblique view of the diamond anvils and gasket arrangement can be seen in figure 1.

To achieve the necessary conditions in a DAC we utilize laser heating to raise the sample temperature to thousands of Kelvins while under high pressures. Direct laser heating is often difficult since many materials lack a strong electronic or vibrational transition that can be coupled to the incoming laser light. A strong absorption to the laser can be a problem as well, since heating at the sample-diamond interface results in the heat being transmitted away from the sample by the diamond. It is also important to have a uniform temperature distribution for the sample volume you wish to probe. Furthermore, a diamond is usually opaque at wavelengths shorter than $350 \mathrm{~nm}$ and many simple molecules have electronic absorptions well into the ultraviolet. Given these restrictions, this experiment requires the fabrication of a target that can absorb laser light sufficiently so that high temperatures can be achieved, but is also partially or wholly transparent so that the our spectroscopic technique (discussed below) can be conducted simultaneously with the laser heating. This target must be relatively inert, be able to withstand very high temperatures, be made small enough to fit inside the miniscule sample volume, and it must leave enough space for the sample to surround the target and act as something of an insulator to the diamond anvils. One of the major goals in this study has become finding a way to do this. Many of the previously developed techniques used for laser heating, which are sufficient for samples that are to analyzed after being quenched from high temperatures, simply can not be applied here.

Heating has been accomplished by focussing a cw laser (Nd:YAG or Nd:YLF) onto a microscopic metal toroid. The toroid is $\sim 20 \mu \mathrm{m}$ thick with an outer diameter of $\sim 80 \mu \mathrm{m}$ and an inner 
diameter $\sim 35 \mu \mathrm{m}$ and fits inside the DAC gasket aperture. This is shown in figure 1. Care must be taken to insure that the toroid is held on the inside aperture wall by a tiny dab of vacuum grease and does not touch either diamond, since the conduction loss will prevent the sample from being heated and might damage the anvils. The toroids are cut out of tungsten or tantalum foil using electric discharge machining (EDM.) The lasers are configured in the $\mathrm{TEM}_{01}$ mode so that the laser profile matches the donut-like shape of the target. The temperature is measured by obtaining the Planck radiation spectrum and fitting it to a blackbody curve. We have already manufactured these toroids and demonstrated that they can be heated while under pressure in a DAC containing nitrogen.

The CARS technique is a third order nonlinear process that requires laser pulses of two different colors (energy) having high peak power (megawatts) to overlap in a sample both spatially and temporally. The difference in photon frequency must be resonant with a Raman active vibration of the molecule. A third color, the signal, is produced by annihilation of two photons of the bluer $\left(\omega_{1}\right)$ color and creation one of the redder $\left(\omega_{2}\right)$ color (see figure 2$)$. The resulting signal is due to the vibrationally resonant and nonresonant part of the third order susceptibility of the analyte. However, as can be seen in figure 2 , the resonant signal dominates the spectrum.

In order to use the CARS technique in a DAC, we require short ( $<200 \mathrm{ps})$ pulses with high peak power. The total energy of the pulses is small so that damage to the diamonds is prevented. Producing the necessary pulses requires a mode-locked Q-switched laser. We have chosen to use a Nd:YAG laser. The Q-switch (rep rate) is typically set to one kilohertz and the mode-locker is set to $\sim 41 \mathrm{MHz}$. The result is a pulse train of approximately 60 to 80 pulses each separated by $\sim 12 \mathrm{~ns}$ being emitted every millisecond. We select one of the pulses from the train and frequency double it to act as $\omega_{1}$ for the CARS experiment. The other pulses are also frequency doubled and synchronously pump two dye lasers. The first dye laser contains no tuning elements so that it can lase broadband. The second dye laser has both a prism and etalon so that it can be narrow band. This allows the second laser to be used for the $\omega_{1}$ source if a low frequency vibration $\left(<600 \mathrm{~cm}^{-1}\right)$ needs to be studied. The pulses from the first dye laser are spatially and temporally overlapped with $\omega_{1}$ inside the toroid hole to produce the CARS signal. The entire vibrational spectrum can be obtained by each pulse pair since the bandwidth of $\omega_{2}$ is typically $\sim 200 \mathrm{~cm}^{-1}$. Carefully choosing the dye and its concentration permits the selection of the central frequency of $\omega_{2}$. The schematic representation of this setup is shown in figure 3. There are two main advantages of CARS over classical Raman spectroscopy. The first is that the signal is directional and comes out well collimated. The second is 
that the signal is pulsed. This permits the signal to be spatially and temporally filtered. The Planck radiation comes out in all direction, just like the signal from classical Raman spectroscopy. Only a simple iris is needed to cut out most of the unwanted background from the sample's incandescence. Also, the signal can be temporally filtered by time gating the CCD detector. The CARS pulses are typically $<100$ ps long, while the Planck emission is continuous. The CARS system has been built.

\section{Summary:}

We have completed construction of a system capable of obtaining spectroscopic information on samples at high pressures and temperatures using diamond-anvil-cells, CARS and laser heating. Microfabrication of metal toroids allow us to heat transparent samples by high-powered lasers. The system is now up and running and we are poised to generate data on nitrogen very soon. Since this project funding ended after 2 years, we were not able to include in this report the results that we had planned to get in year 3 . We are seeking programmatic funding sources to continue this project. 


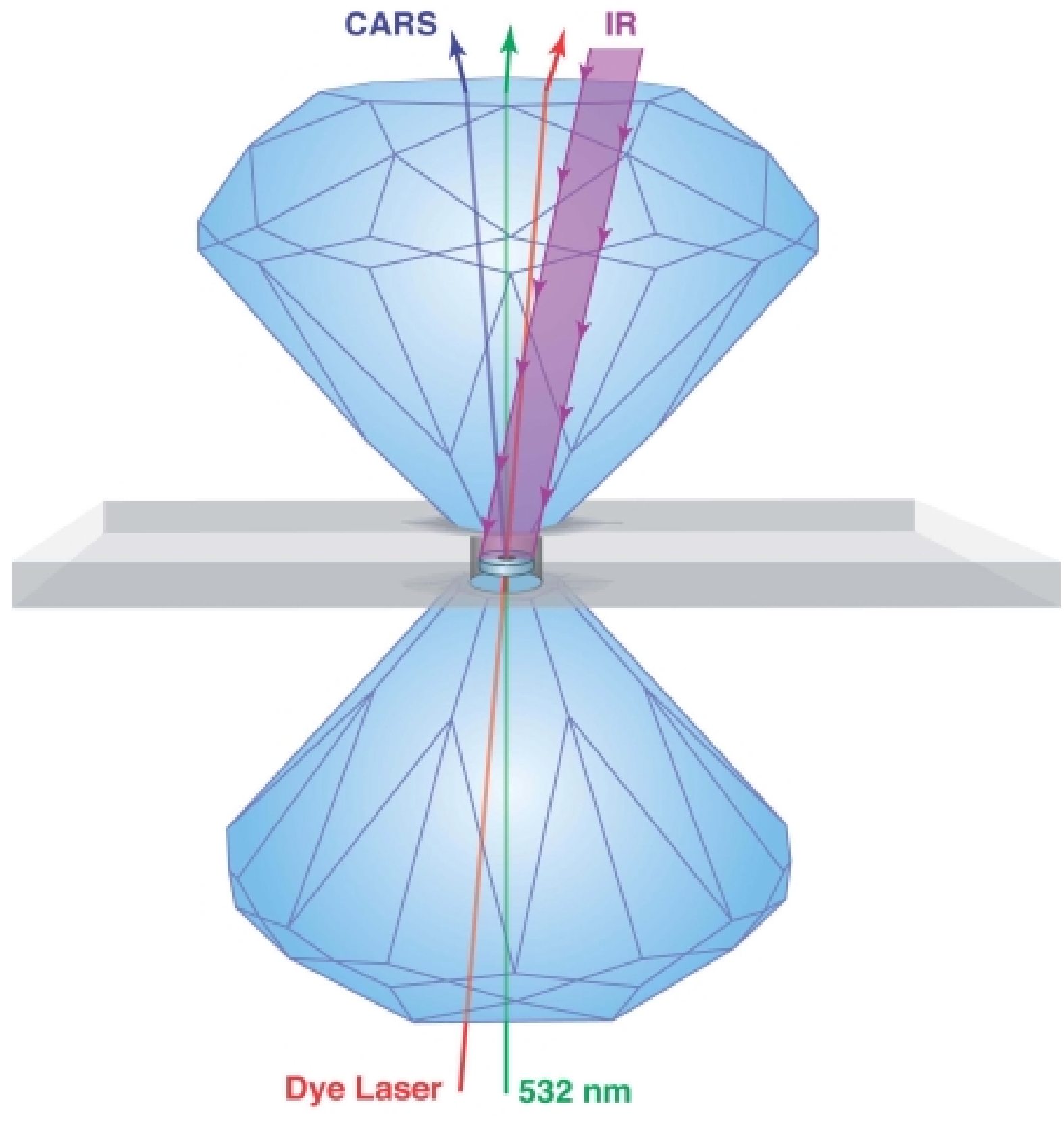

Figure 1: Diamond anvils, gasket and laser heating target arrangement. The laser beams for both the heating and CARS experiment are shown. 

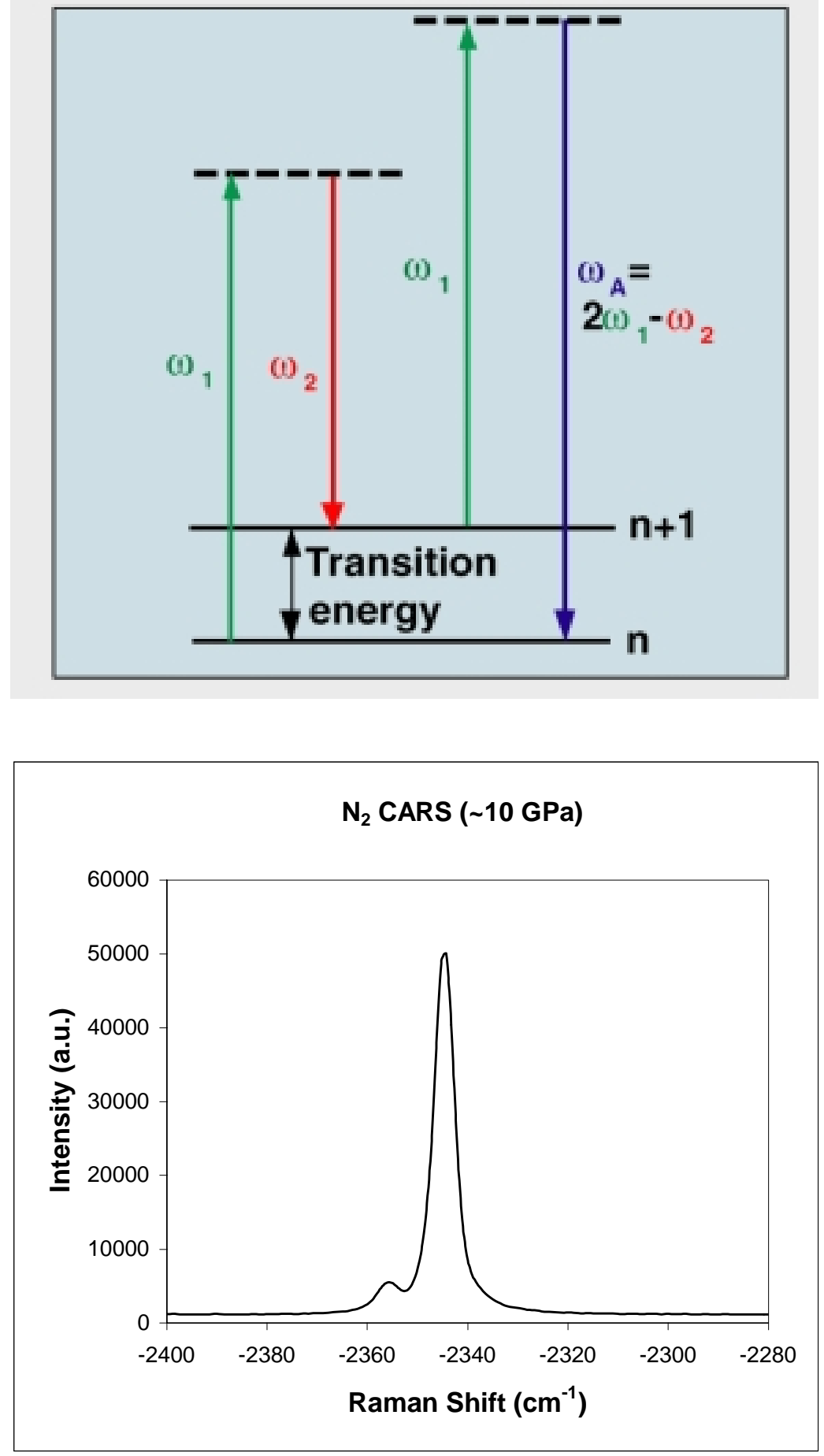

Figure 2:

upper panel: Schematic representation of the CARS process. lower panel: typical CARS spectrum at ambient temperature 


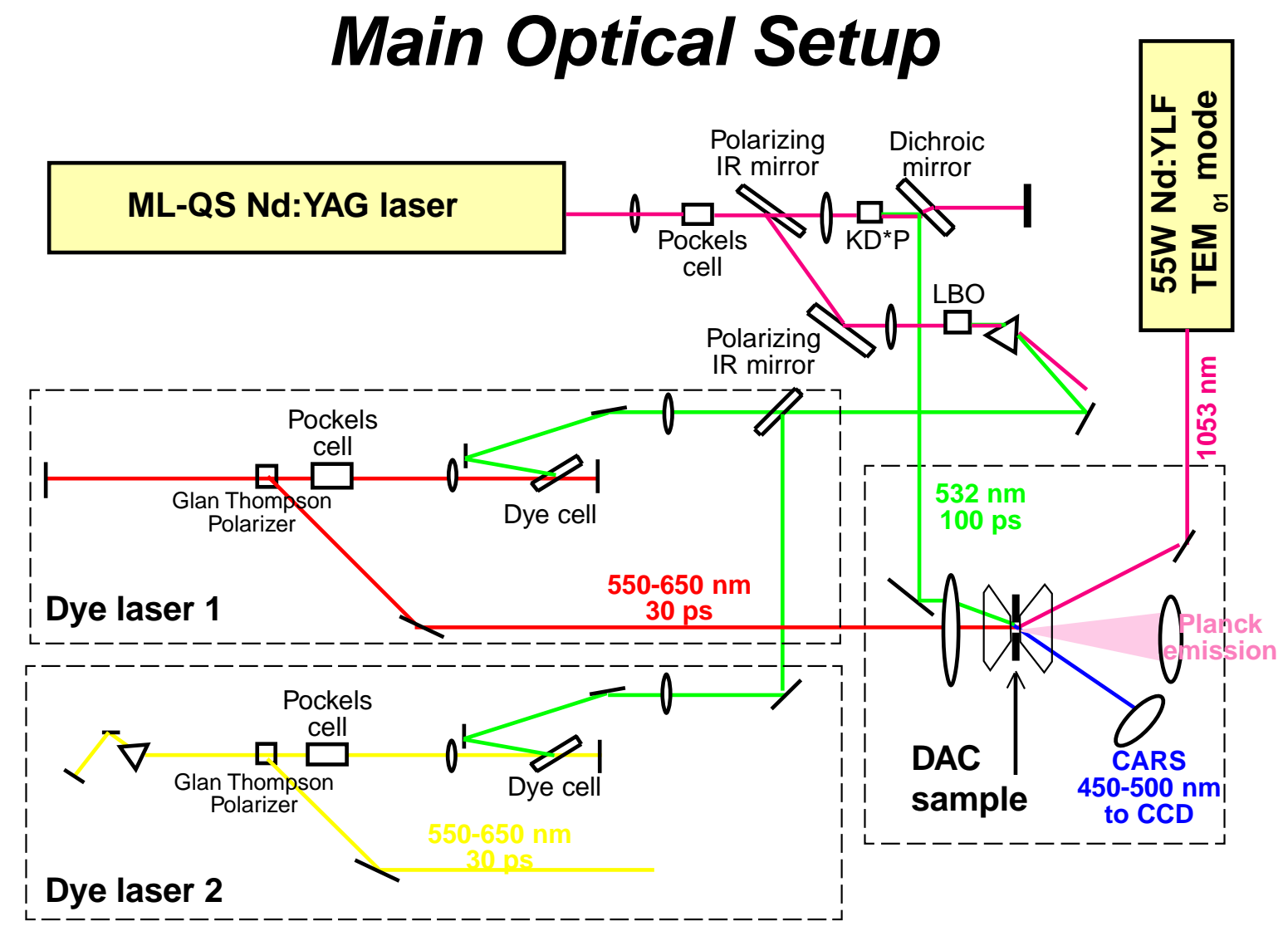

Figure 3: Schematic layout of the experiment. 


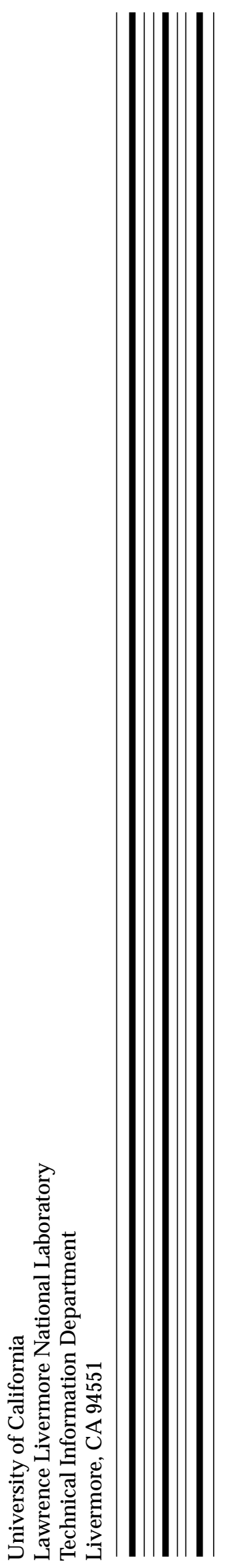

